\title{
Consortium Blockchain based Reputation Incentive Mechanism for Recommendation System
}

\author{
Guo Sun ${ }^{1}$, Tingting Zhao ${ }^{1}$, Qingyi $\mathrm{Ye}^{1}$, Chuntang $\mathrm{Yu}^{1}$, and Xia Feng ${ }^{2}$ \\ ${ }^{1}$ School of Computer Science and Communication Engineering, Jiangsu University, Zhenjiang, China \\ ${ }^{2}$ School of Automotive and Traffic Engineering, Jiangsu University, Zhenjiang, China
}

\begin{abstract}
Recommendation systems have been widely used in many e-commerce services, but it is difficult to gather enough participants to supply their recommendations. Moreover, participants in the system may make malicious recommendations, which will affect the accuracy of recommendation results. In order to provide better recommendation service for users, incentive mechanisms are needed to attract more participants in recommendation and curb their malicious behaviors. In this paper, we propose a consortium blockchain based reputation incentive mechanism for recommendation systems(CRIM). Firstly, the monetary rewards are used to attract participants and motivate them to take part in the recommendation. Secondly, we design the incentive mechanism with reputation which is attached to the rewards. Honest participants will gain more rewards while malicious participants will be penalized. Meanwhile, we adopt the Stackelberg game to maximize the utility of participants, and prove that the mechanism can reach a unique Nash equilibrium. Thirdly, the decentralization and immutability of blockchain can guarantee the credibility and security of the stored data, thus ensuring the openness and transparency of the recommendation. Finally, we implement the system for education resources recommendation and conduct experiments, and the results demonstrate that our incentive mechanism is effective and has significant performance when compared with other incentive mechanisms.
\end{abstract}

Index Terms-Consortium blockchain, reputation incentive, game theory, recommendation system,

\section{INTRODUCTION}

$\mathbf{N}$ OWADAYS, recommendation systems are widely applied in various areas of life, such as Amazon, eBay, and Netflix. They all use recommendation technology in their systems to estimate potential customer preferences and recommend relevant products to users. These applications provide convenience for users to quickly find the information they need in massive amounts of data. The effectiveness of recommendation systems depends heavily on the ability to extract historical data information precisely from users at a large scale. However, recommendation engines are generally architected on centralized servers which may suffer from single point of failure and is vulnerable to be attack. The risk of user privacy data leakage is huge. In addition, the key to recommendation is to make users trust and accept the recommendation results.

Blockchain has established a new trust model for global interconnection and brought new opportunities for the resolution of pain points in various industries due to its features of decentralization and immutability [1].In recent years, it has attracted much attention from academia and industry [2], [3]. The work of [4] proposes some of the current blockchain applications for education and discusses its benefits and challenges. Although blockchain has brought many innovations to the education industry, there are still some potential drawbacks that have not been solved. The work of [5] analyzes the pain points faced by the field of higher education, and proposes a fair recommendation system for educational resources based on blockchain technology to achieve fair and credible recommendation of educational resources. The recommendation system

Manuscript received November 5, 2021; revised January 11, 2022. Corresponding author: Qingyi Ye (email: yqy_city@163.com).

*Extention: The part of this work was presented in 2021 International Conference on Networking and Network Applications (NaNA). brings together teachers, experts and other people who have in-depth knowledge of educational resources, and recommends through smart contracts.

However, the blockchain-based decentralized recommendation system still faces several problems. On the one hand, the accuracy of the recommendation results depends on the number of participants in the system, but it is difficult to gather enough participants to take part in recommendations. On the other hand, there may be malicious participants in the system, and their dishonest recommendations will affect the final results. These problems greatly affect the accuracy of the results and the usability of the recommendation system. In such a system which needs high degree of participation, the introduction of an incentive mechanism can motivate enough participants to continuously take part in the recommendation task, thus ensuring the long-term stability of the system. Therefore, how to design a reasonable incentive mechanism to motivate participants to actively take part in the recommendation task and honestly make recommendations is an important issue of the paper.

To address the problems mentioned above, this paper proposes a consortium blockchain based reputation incentive mechanism for recommendation system by considering the current popular incentive mechanism and blockchain-based incentive mechanism. Meanwhile, using blockchain technology to reconstruct the underlying foundation of the recommendation system can ensure the privacy of users, the safe operation of the system, and the credibility of data. By using the Stackelberg game to distribute rewards, the paper motivates participants to actively take part in the recommendation task, ensuring the number of participants and improving the quality of the recommendation results. Moreover, the accumulation of rewards from honest recommendations can make users addicted to the cooperative behavior, thus motivating their 
honest participation. Finally, we analyze its equilibrium at which the utilities of the user and recommendation participants are optimized. The main contributions of this paper are as follows:

- We propose a recommendation system on the consortium blockchain, which can achieve open and transparent recommendations with multi-party participation.

- We introduce a reputation incentive mechanism to motivate participants to take part in the recommendation system and make honest recommendations, which ensures the accuracy of recommendation results.

- We design the reputation incentive mechanism to maximize participants' utility through a two-stage Stackelberg game, and prove that the mechanism can reach a unique Nash equilibrium.

- We have implemented the designed recommendation system in the field of higher education and the simulation experiments demonstrate the significant performance of our proposed incentive mechanism.

\section{RELATED WORK}

Incentive mechanism is an crucial issue in the study of crowdsensing applications. Its need for a large number of nodes to participate is very similar to the recommendation scenario in this paper. Next, we will analyze the existing incentive mechanisms applied to the crowdsensing applications to explore the incentive mechanism applicable to the recommendation system in this paper.

Yang et al. [6] proposed two types of incentive mechanisms for crowdsourcing systems from the perspective of agent platforms and mobile users respectively. The platform-centric mechanism is based on the Stackelberg game, which assumes that the platform has absolute control over the total amount of payments made by users, and that users can only participate in the system to increase their own revenue by adjusting their strategies. The user-centric incentive mechanism utilizes an auction-based scheme, which maximizes the utility of the participants. A game-theoretic analysis of bitcoin [7] by Kroll et al. argues that honest strategies constitute a Nash equilibrium (NE) in the sense that the system will operate stably if all parties act in accordance with its incentives. Lee and Hoh [8], [9] design a reverse auction-based virtual dynamic price incentive mechanism for participation points (RADP-VPC), which aims to minimize and stabilize the platform cost while maintaining participation. The mechanism not only reduces the incentive cost of retaining the same number of participants, but also improves the fairness of incentive distribution. Duan et al. [10] designed a threshold revenue model for service providers using the Stackelberg game.

Reputation mechanisms can provide incentives to prevent malicious behavior [11], [12]. Zhao et al. [13] propose a new blockchain-based fair payment to study cyber-physical systems(CPS) in which a reputation system is applied to allow subscribers to evaluate published events and tag publishers based on reputation. Sharples and Domingue [14] propose a blockchain-based permanently distributed knowledge effort record that instantiates and democratizes educational reputation beyond academia. Burak et al. [15] combine centralized reputation-based evaluation with collaborative reputation values based on votes.

The existing blockchain-based incentive mechanism is rapidly growing due to the advantages of the blockchain technology itself. In TrueBit [16], users upload the tasks they need to perform, participants can perform them for them and earn reward money, and others verify the correctness of the execution results. Etherium rewards honest participants and punishes malicious ones by using smart contracts as the ultimate arbiter. Fraudsters are financially penalized, so in the vast majority of cases, participants will perform the task honestly and provide correct results. The chain nodes do not require complete verification, thus reducing the amount of computation on the chain. He Y et al. [17] propose a blockchainbased real incentive mechanism that uses cryptocurrencies such as Bitcoin to incentivize users to cooperate. Our previous work [18] introduced a reputation incentive mechanism into a recommendation scheme, which can effectively increase the user's system participation and at the same time resist some malicious collusion behavior.

Although the above research work has made some progress, there are still some limitations. These studies are good at ensuring user participation within a certain number of rounds through some incentive mechanisms, but lack consideration of the long-term operation of the system. With the increment of system operation time, the system operation effect after a period of time is often lower than expected because of the existence of diminishing marginal utility. Therefore, for the problem that the accuracy of recommendation results relies on the number of participants, we synthesize the advantages of these related works and design an incentive mechanism applicable to the scenario of consortium blockchain-based recommendation systems in this paper. Meanwhile, we consider the idea of crowdsensing and use the Stackelberg game to motivate the degree of participation. For the problem that participants may be evil, we designed the incentive mechanism based on reputation and distribute rewards depend on the efforts to prevent malicious behavior. On this basis, we introduce a growth function to accelerate the accumulation of rewards and penalties to induce users to be addicted to cooperative behavior, thus ensuring their long-term participation. Together with, the innate advantage of blockchain technology in incentive mechanism, this paper designs a consortium blockchain based reputation incentive mechanism for recommendation system.

\section{PRELIMINARY}

\section{A. Blockchain Technology}

The emergence of blockchain technology has brought about a trust revolution and is being used across industries to solve trust issues. Blockchain was first proposed [19] by "Satoshi Nakamoto" in the Bitcoin white paper "Bitcoin: A Peer-toPeer Electronic Cash System”. Blockchain technology is a combination of distributed data storage, peer-to-peer transmission, consensus mechanisms, and cryptographic algorithms. It uses chained data structures to validate and store data, cryptography to secure data transmission and access, and smart contracts to program and process data. In recent years, it has 
attracted a great deal of attention from academia and industry [20], [21]. Various industries have begun to pay attention to blockchain technology, actively exploring and using the technology to solve industry problems and promote industry innovation and development [22].

As a kind of permission blockchain, the consortium blockchain can decide the degree of openness to the public according to the application scenario. Its network is jointly maintained by consortium institutions and nodes are accessed through the gateway nodes of consortium institutions, so it is suitable for the storage, management, monitoring and auditing of dynamic data by multiple institutions in the context of computer industry, and the incentive mechanism of data operation among various entities is also a current research hotspot.

\section{B. Stackelberg game}

In recent years, with the development of computer industry, many experts and scholars in computer networks have started to rely on game theory to solve the problem of "maximizing user's income" in the network under certain rules. A reasonable game theory strategy can guide users to choose the right strategy to ensure that they get the maximum benefit, and the benefit of each individual in the network can be maximized at the same time.

The Stackelberg game [23], [24] is a master-slave game that describes a game between a leader and a follower, in which the user is the leader who can publish requirements and formulate strategies, and the recommendation participant is the follower who can choose to participate in the consortium organization as a node according to the leader's strategy to make recommendations. The blockchain-based system platform is responsible for the data interaction between users and participants and the execution of the recommendation process. The main idea is a two-stage game. In the first stage, users publish tasks; in the second stage, participants make strategies to choose whether to participate in the recommendation. The recommendation is completed when the number of participants exceeds the minimum number specified by the system, after which the system receives revenue and the participatants receive rewards. Otherwise, this recommendation task will not have any revenue.

\section{SYSTEM MODEL}

In this paper, we propose a recommendation system model with reputation incentive mechanism, which can motivate participants to take part in the recommendation system and complete the recommendation tasks honestly. Participants will get different monetary rewards according to the recommendation behaviors. The system contains three roles:

Users: The user in the recommendation system is the publisher of the recommendation task, who publishes his recommendation requirements to the system platform, and gets the final recommendation results from the platform after the system completes the recommendation.

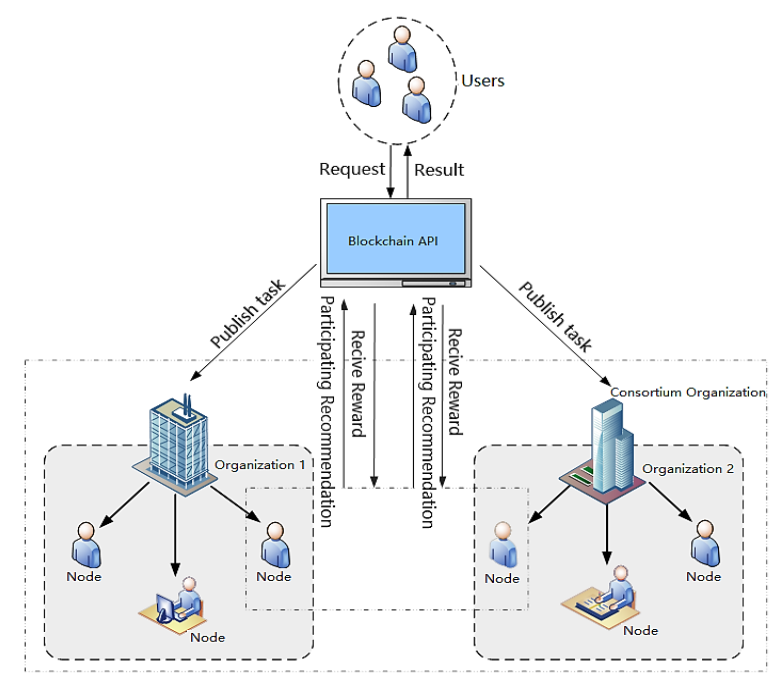

Fig. 1: Recommendation system model with reputation incentive mechanism on consortium blockchain

Participants: The participants in the recommendation system come from multiple authorized organizations or institutions, and are mainly responsible for completing the recommendation process in response to the recommendation requirements proposed by users and feeding the recommendation results to the platform.

System platform: The system platform provides data interaction between users and participants. On the one hand, it is responsible for accepting users' requirements and packaging them into recommendation tasks, and on the other hand, it is responsible for publishing recommendation tasks to participants and distributing rewards.

In the system model diagram shown in Fig.1, multiple organizations can join together to build a blockchain network, and the recommendation participants in the organizations are the blockchain nodes authorized by the consortium blockchain certification. First, users will join the system and send recommendation requirements to the system platform, which publishes recommendation tasks to participants in the blockchain system according to the users' recommendation requirements and adopts a incentive mechanism to attract more nodes to participate in this recommendation. Then, the nodes participating in the recommendation will invoke the recommendation smart contract deployed on the blockchain to execute the recommendation. After receiving the recommendation result from the blockchain network, the system returns it to the user and distributes the rewards to the participating nodes. It is worth mentioning that the process of participants making recommendations and receiving system rewards by invoking smart contracts can be regarded as a transaction, which will be packaged and recorded on the blockchain after being verified by miners.

In the above recommendation system model, we introduce a reputation incentive mechanism, which should require the following design principles.

- Incentives are designed mainly to enable better recommendation results, and it is crucial to ensure the 
number of participants. Therefore, participants should be adequately rewarded for their participation, at least not below their cost price.

- The operation of the system requires the long-term continuous participation of nodes. Thus higher, even incremental rewards should be ensured for honest participants involved in the recommendation, and the probability of receiving penalties should be small.

- The incentive mechanism should be designed in such a way that it can effectively curb malicious nodes, because the decline of the mechanism's credibility will affect the recommendation results. Therefore, the mechanism should focus on fair and transparent distribution of rewards.

\section{REPUtATION INCENTIVE MECHANISM}

The goal of incentive design is to motivate participants and maximize their utility. In this chapter, we model the proposed reputation incentive as a Stackelberg game to determine how to optimize the utility between the participants and users.

\section{A. Incentive model}

According to the above design principles of reputation incentive mechanism, the system platform must effectively incentivize the participants to ensure that enough participants participate in the recommendation in order to make the recommendation requirements submitted by users get more satisfactory results and thus improve the accuracy of the recommendation results. The optimal utility of the participants comes from the optimal distribution of rewards, so our proposed reputation incentive mechanism consists of two parts: node reputation assessment and reward distribution. The incentive model will be introduced first in the following.

Assuming that the set of nodes involved in the recommendation $N=(1, \ldots, n)$, the total number of $N$ is the nodes within all consortium organizations. To the node $i \in N$, its participation cost $C i>0$ and is independent and identically distributed with mean $\mu$ and cumulative probability distribution function $F(\cdot)$. The user should give a reward $R$ while publishing a recommendation requirement. The system successfully completes this recommendation task if the current recommendation request involves at least $\alpha$ nodes to make the recommendation. The system receives $V$ revenue and the participating nodes receive the corresponding rewards. If this recommendation task is not successfully completed, no revenue will be earned. The process is shown in Fig.2.

Step 1: The user publishes a task request $T$ to the system and shows the reward $R$.

Step 2: System publishes task $(T, R, \alpha)$, where $R$ is the total reward to all participants and $\alpha$ is the threshold number of participating nodes required for this recommendation.

Step 3: The nodes in the consortium organization choose whether to participate in this recommendation.

Step 4: If the node decides to participate in the recommendation, it needs a certain deposit of $D$, which will be returned after completing the recommendation. But the deposit will be deducted if the node participates maliciously.

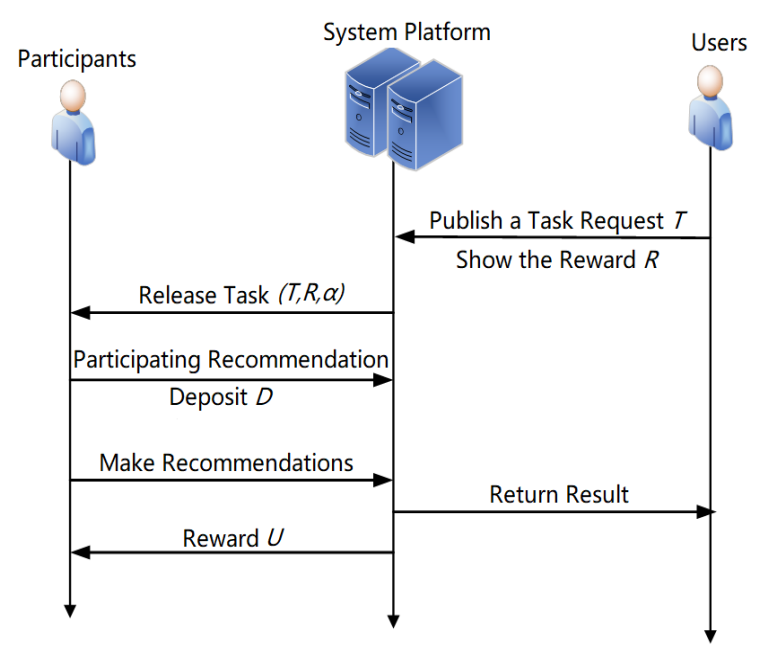

Fig. 2: Flow chart of recommendation system with reputation-based incentive mechanism

Step 5: Within a certain period of time, if the number of participating nodes reaches a threshold value,the recommendation will complete successfully.

Step 6: The system will return the recommendation results to the user and distribute the rewards to the participants according to their efforts.

\section{1) Node reputation assessment}

First of all, for a new authorized node to join the blockchain network, it needs to pay a certain deposit, and the system will give an initial reputation value according to the amount of deposit it pays. The deposit mechanism can help the newly joined nodes to improve the reputation level quickly, and the more the deposit the higher the reputation level. If a node has malicious behavior, the deposit will be deducted. Suppose the initial reputation value of the participant is $D$, then.

$$
D=\frac{D_{i}-D_{\min }}{D_{\max }-D_{\min }}
$$

Where $D_{i}$ denotes the deposit paid by nodes, $D_{\max }$ denotes the maximum amount of the deposit paid by all nodes, and $D_{\min }$ denotes the minimum amount of the deposit paid by all nodes.

Second, the positivity of node $P$ is related to the participation enthusiasm of nodes in the recommendation tasks in the blockchain network and can be expressed as:

$$
P=\frac{n_{t s}}{n_{t}}
$$

Where $n_{t}$ denotes the number of recommendation tasks published by the system since the node joined the system, $n_{t s}$ denotes the number of recommended tasks accepted by the node.

Next, the credibility $L$ of node is related to the completion of the node's recommendation tasks in the blockchain network and is weighted by two components, expressed as follows.

$$
L=(1-\delta) \frac{n_{s}}{n}+\delta \frac{\sum_{i=1}^{n} U_{i}}{\sum_{i=1}^{n} U_{s i}}
$$


Where $n$ denotes the number of times a node accepts a recommendation task, and $n_{s}$ denotes the number of times the node completes the recommendation honest. The $U_{s i}$ denotes the amount of reward that should be obtained by the node's honest completion of an accepted recommendation task, and $U_{i}$ denotes the actual reward amount obtained by the node in an recommendation task. $\delta$ is a coefficient, and the larger $\delta$ is, the more importance is attached to the failure of recommendations with large rewards; if $\delta$ is equal to 0 , it means that only the number of honest recommendations is emphasized.

Finally, in this paper, the node reputation value $T$ is calculated by three dimensions: node's initial reputation value, positivity and credibility.

$T=\gamma\left((1-\delta) \frac{n_{s}}{n}+\delta \frac{\sum_{i=1}^{n} U_{i}}{\sum_{i=1}^{n} U_{s i}}\right)+\beta \frac{n_{t s}}{n_{t}}+\frac{D_{i}-D_{\min }}{D_{\max }-D_{\min }}$

Where $\gamma$ is the weight of the node's credibility and $\beta$ is the weight of the node's positivity.

\section{2) Reward distribution}

The recommendation system contains two roles. One is the participant, who makes resource recommendations, pays a certain deposit $D_{i}$ and receives a reward $U_{i}$ after the recommendation is completed; the other is the user, who publishes the recommendation and pays a certain reward $R$ to all the participants. After determining a set $P=(1, \ldots, p)$ of nodes that successfully participated in a recommendation, the system platform needs to calculate the reward $P_{i}$ that should be paid for each participating node $U_{i}$. If the node is not in this set, then $U i=0$; otherwise, $U_{i}>0$ and $U_{i}$ should be no less than the participant's cost price $C_{i} . U_{i}$ may be different for different participants. In order to motivate nodes to make honest recommendations, if the recommendations of some participants are consistent with the final results, nodes that are considered as credible participants. These participants will receive higher additional rewards consist of deducted deposits from malicious participants.

Definition of credible node: If the reputation value $T$ of a consortium node exceeds the credit $\operatorname{threshold}(\tau)$, it is considered as a credible participant and can participate in this recommendation.

Definition of malicious node: If the reputation value $T$ of a consortium node is less than the malicious threshold $(\sigma)$ after the current recommendation, it is considered as a malicious participant.

In order to prevent the situation that some malicious nodes do not care about the deposit and reward money for participating in the recommendation task and only perform malicious behaviors, we introduce a growth function $f(T)=\rho^{T}$ based on the reputation value of nodes. Where $\rho$ is a factor, $0<\rho<1$. The higher the reputation value of a node, the lower the deposit he should pay before participating in the recommendation task. In contrast, if the node has malicious behavior many times, his reputation value becomes lower and lower, and the deposit paid each time he participates in the recommendation will be higher.
Finally, assume that there are $P$ nodes willing to join this recommendation task, where the number of credible participants is $e$, and there are $A=(1, \ldots, a)$ nodes among the honest participants whose recommendation results agree with the final result and $B=(1, \ldots, b)$ nodes whose recommendation results do not agree with the final result. The number of malicious participants is $M=(\mathrm{P}-e)$. Therefore, the utility function obtained by each participating node after deducting the cost is as follows.

$$
U_{i}=\left\{\begin{array}{r}
\frac{R}{m}+\sum_{i=1}^{m} f\left(T_{i}\right) D_{i} / a-c_{i}, i \in P \text { and } i \in A, \\
\frac{R}{m}-c_{i}, i \in P \text { and } i \in B, \\
-c_{i}, i \in P \text { and } i \in M .
\end{array}\right.
$$

When $i \in P$ and $i \in A$,the node is credibly recommended and the recommendation result is consistent with the final result, so the node receives an additional reward $\sum_{i=1}^{m} f\left(T_{i}\right) D_{i} / a$ in addition to normal rewards; when $i \in P$ and $i \in B$, the node receives a reward for normal recommendation; when $i \in P$ and $i \in M$, the node makes a malicious recommendation, so it does not receive a reward and even loses its cost and margin.

\section{B. Two-Stage Stackelberg Game Formulation}

Based on the reputation incentive mechanism described in this chapter, we can formulate the mechanism as a two-stage Stackelberg game.

In the first stage, the user publishes a requirement in the system and gives the amount of reward to incentive the participants to make a recommendation for the task. Since no participant would join a recommendation without gain, our reward money is more than the cost.

In the second stage, each participant makes his or her own strategy and decides to participate in different recommendation tasks to try to maximize the utility. Assuming $h=$ $\left(h_{1}, \ldots, h_{n}\right)$ denotes the set of strategies for the participants. In this incentive mechanism, the user is the leader and the participant is the follower. The goal of the mechanism is to find a Nash equilibrium. In this case, the user can maximize his utility based on the participants' receipt and completion of the recommendation tasks, while the participants maximize their utilities through the reward distribution designed in the mechanism. It is represented as follows.

In the first stage:

$$
\text { Maximize } U_{\text {user }}(R), \quad \text { Subject to } R>0
$$

In the second stage:

$$
\text { Maximize } U_{N}^{h}, \quad \text { Subject to } h_{i}>0
$$

According to the previous definition, $N=(1, \ldots, n)$ denotes the set of nodes involved in the recommendation; $R$ denotes the reward given by the user when publishing the recommendation request. In addition, $U$ denotes the utility gained by users or participants; $h_{i}$ denotes the strategies made by participants. 


\section{Equilibrium Analysis for incentive mechanism}

In this section, we analyze the optimal strategies of the participants and the utility maximization of the users, and argue for a Nash equilibrium of this incentive mechanism. In the second stage of the game of this mechanism, the rewards of all recommendation tasks are known, and participants compete with each other to maximize their utility by choosing their respective strategies, which can be considered as a Recommendation Game(RG).

For each recommendation task, the user announced a total reward $R>0$ to incentive participants to join the recommendation. Each participant decides his strategy of recommendation based on the reward it gives. The strategy of any participating node $i \in N$ is represented by $h_{i}>0$. Specifically, if $h_{i}=0$, it indicates that the node will not participate in the recommendation task. Assume that the participation cost of node $i$ is $c_{i} h_{i}$, where $c_{i}>0$ is its cost in a recommendation task. The reward received by node $i$ is proportional to $h_{i}$. Then the utility of a node can be defined as the difference between reward and cost:

$$
U_{i}=\frac{h_{i}}{\sum_{j \in N} h_{j}} R-c_{i} h_{i}
$$

Let $h=\left(h_{1}, \ldots, h_{n}\right)$ denotes the set of strategies for the participating nodes. Let $h_{-i}$ denote the strategy profile excluding $h_{i}$. Therefore, we record $\boldsymbol{h}=\left(h_{i}, h_{-i}\right)$. Before demonstrating the Nash equilibrium of the Recommendation Game(RG), we first give a definition.

Definition 1 (Nash Equilibrium, NE). A set of strategies $\left(h_{1}^{\prime}, h_{2}^{\prime}, \ldots, h_{n}^{\prime}\right)$ is a Nash Equilibrium of the Recommendation Game if for any node $i, U_{i}\left(h_{i}^{\prime}, h_{-i}^{\prime}\right) \geq \bar{U}_{i}\left(h_{i}, h_{-i}^{\prime}\right)$ for any $h_{i} \geq 0$.

Theorem 1. A Nash Equilibrium in the Recommendation $\operatorname{Game}(R G)$ exists.

Proof: Based on the definition of NE, every node is making its best strategy in a NE. To study the best strategy of node $i$, we compute the derivatives of $U_{i}$ with respect to $h_{i}$ :

$$
\begin{gathered}
\frac{\partial U_{i}}{\partial h_{i}}=\frac{1}{\sum_{j \in N} h_{j}} R-\frac{h_{i}}{\left(\sum_{j \in N} h_{j}\right)^{2}} R-c_{i} \\
\frac{\partial^{2} U_{i}}{\partial h_{i}^{2}}=-\frac{2 R \sum_{j \in N \backslash\{i\}} h_{j}}{\sum_{j \in N} h_{j}}<0
\end{gathered}
$$

Since the second-order derivative of $U_{i}$ is negative, the utility $U_{i}$ is a strictly concave function with $h_{i}$. Therefore, given any $R>0$ and any strategy $h_{-i}$ of the other nodes, the best strategy $\beta_{i}\left(h_{-i}\right)$ of user $i$ is unique, if it exists. Accordingly, the Nash Equilibrium exists.

Further, by setting the first derivative of $U_{i}$ to 0 , we have:

$$
\frac{1}{\sum_{j \in N} h_{j}} R-\frac{h_{i}}{\left(\sum_{j \in N} h_{j}\right)^{2}} R-c_{i}=0
$$

Solving for $h_{i}$, we obtain:

$$
h_{i}=\sqrt{\frac{R \sum_{j \in N \backslash\{i\}} h_{j}}{c_{i}}}-\sum_{j \in N \backslash\{i\}} h_{j}
$$

If the right-hand side of (10) is positive, it is also the best strategy of node $i$, due to the concavity of $U_{i}$. If the right-hand side of (10) is less than or equal to 0 , then node $i$ does not participate in the recommendation task. Therefore:

$\beta\left(h_{i}\right)=\left\{\frac{0}{\sqrt{\frac{R \sum_{j \in N \backslash\{i\}} h_{j}}{c_{i}}-\sum_{j \in N \backslash\{i\}} h_{j}}}\right.$

if $R \leq c_{i} \sum_{j \neq i \cap j \in N} h_{j}$ otherwise

In conclusion, $h^{\prime}$ is an NE of Recommendation Game(RG).

Theorem 2. The Nash Equilibrium in the Recommendation Game $(R G)$ is unique.

Proof: First, we assume that there exists one node $i \in$ $N$ whose $h_{i}^{*} \neq h_{i}^{\prime}$, but it also satisfies $U_{i}\left(h_{i}^{*}, h_{-i}^{\prime}\right) \geq$ $\bar{U}_{i}\left(h_{i}, h_{-i}^{\prime}\right)$ for any $h_{i} \geq 0$.

Then, if the reward $R \leq c_{i} \sum_{j \neq i \cap j \in N} h_{j}^{*}, h_{i}^{*}$ have to be 0 . But $h_{i}^{*}=0$ is contradict with the fact of $h_{i}^{*}>0$. Meanwhile, reminding that (6) is a concave function and it reaches the maximum when $h_{i}=h_{i}^{\prime}$. So, $U_{i}\left(h_{i}^{*}, h_{-i}^{\prime}\right)<\bar{U}_{i}\left(h_{i}^{\prime}, h_{-i}^{\prime}\right)$, which is contradict with $U_{i}\left(h_{i}^{*}, h_{-i}^{\prime}\right) \geq \bar{U}_{i}\left(h_{i}, h_{-i}^{\prime}\right)$ for any $h_{i} \geq 0$.

In conclusion, the $\mathrm{NE}$ of $\operatorname{Recommendation} \operatorname{Game}(\mathrm{RG})$ is unique.

\section{DESIGN AND IMPLEMENTATION OF RECOMMENDATION SYSTEM}

In this chapter, we design and implement a higher education resources-oriented recommendation system on consortium blockchain. The system establishes an educational consortium blockchain through the joint multiple education organizations such as universities and institutions. The purpose of the system is to provide users with effective education resource recommendation, for example, course recommendation, teacher recommendation and school recommendation. The operating system is Ubuntu 64-bit system based on the fabric development environment.

The system designed in this paper is a consortium blockchain-based recommendation system for education resources. There are two roles in the system: ordinary users and consortium nodes. After registering and logging into the system, you can either register as ordinary users to publish recommendation tasks; or participate in the recommendation process as consortium nodes. The functions of the ordinary user side mainly include task publishing, task progress inquiry, result inquiry,and result feedback. The functions of the consortium node side mainly include participation recommendation and reward distribution. The details are as follows.

Fig. 3 shows the homepage of the recommendation system, which contains two search boxes, a publish task button, and a task list. Users can search for recommendation tasks in the task list according to the task name and reward money. The task list 


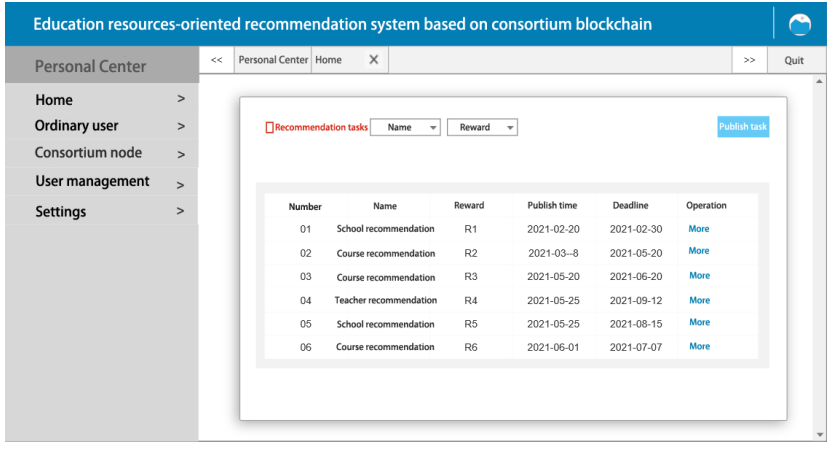

Fig. 3: Recommendation system's homepage

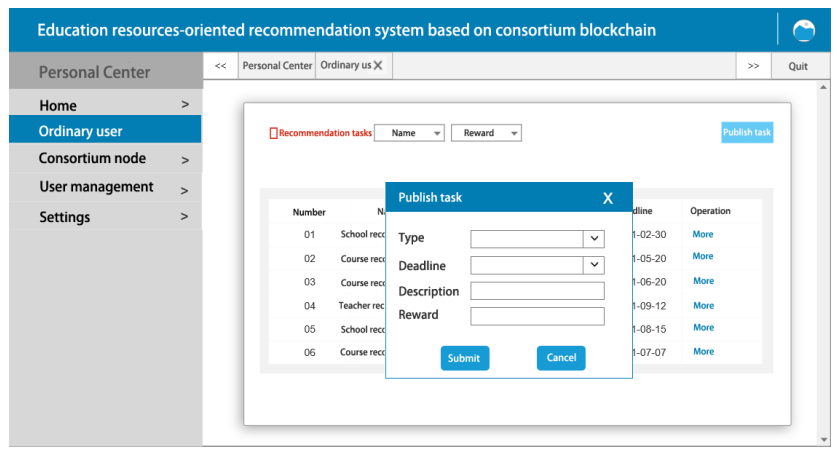

Fig. 4: Recommendation system's task publishing interface

includes: number, name, reward, posting time, deadline, and action. Clicking on a task name will give you a more detailed description of the task. The task number is sorted by the time the task was published. The task publishing time and deadline must be clear and the user can check the task progress status in More. The figure shows the list of unfinished or ongoing tasks, and consortium nodes can choose to participate or not to participate in the recommendation according to their interest or the amount of reward. The user can post a task by clicking the Publish Task, and the published task will be added to the task list.

Fig.4 shows the user task publishing interface of the recommendation system. When submitting a task, the user needs to fill in the task type, task reward, deadline,and task description. The system will generate a task based on the submitted information and publish it to the consortium blockchain network. Consortium nodes choose whether to join or join which task according to the list of tasks.Users can query the task progress and whether the transaction is successfully carried out. If the task is not successful, users can change the reward value and republish it; if the task is successfully carried out and the recommendation result is derived, users can check the recommendation result through the Result Query.

Fig.5 shows the participation interface of the consortium nodes of the fair recommendation system, which mainly includes the participation recommendation module and the reward distribution module. In the participation recommendation module, participants can view the number of recommendations they have participated in and their historical average recommendation success rate.Besides, they can view the rec-

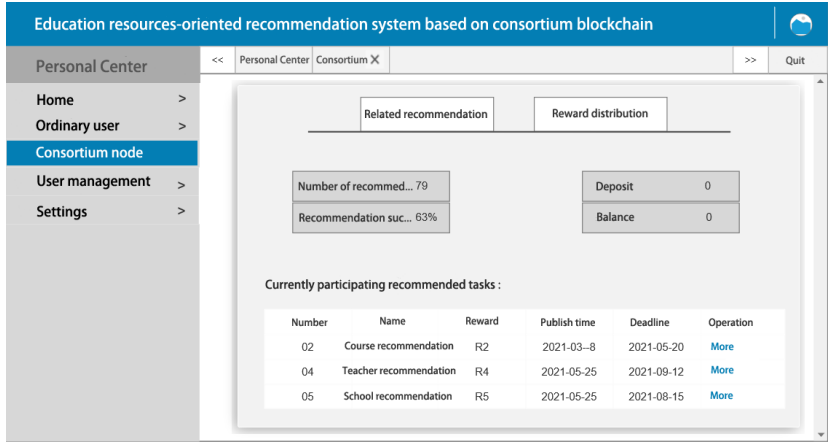

Fig. 5: Recommendation system's consortium node interface

ommendation tasks they are currently participating in and the status of the recommendation tasks in a list. In the reward distribution module, there are two parts: margin and balance. The margin section is generally the unreturned margin, which includes the margin for unfinished recommendation tasks and the margin deducted for malicious recommendations by nodes. The balance section is the reward money obtained from recommendations. Users will transfer different amounts of reward money to the balance account of participants according to the different performances of participating nodes.

\section{SECURITY AND PERFORMANCE ANALYSIS}

\section{A. Security Analysis}

This chapter focuses on the proposed incentive mechanism(CRIM), analyzes the security of the blockchain system, how to achieve security to avoid attacks under the following various collusion attacks, and analyzes the robustness of the system.

\section{1) Security of blockchain systems}

There is no third party presence in the blockchain system, and users and participants execute recommendation transactions on the system platform through smart contracts. Rules for task publish, reward and node assessment are already deployed in advance in the smart contract. Once the smart contract is triggered by the specified behavior, the predefined functions will be executed automatically. The security is guaranteed while the blockchain system also achieving openness and transparency in the transaction processing.

Meanwhile, on-chain miners add new blocks to the blockchain through a consensus mechanism. In the consortium blockchain, each authorized validation node independently validates the transaction, and the packaged new blocks are also broadcast on the network for other nodes to validate. This enhances the security of the system while ensuring the data on the blockchain is tamper-proof, as an attacker who needs to tamper with the blockchain has to pay a huge computational cost. In addition, the access mechanism of the consortium blockchain itself can prevent denial-of-service attacks.

\section{2) Analysis of anti-collusion}

This chapter considers 3 types of collusion attacks.

Collusion attack among participating nodes. Suppose multiple participants launch a collusion attack in this recommendation, causing the recommendation result to be transformed 


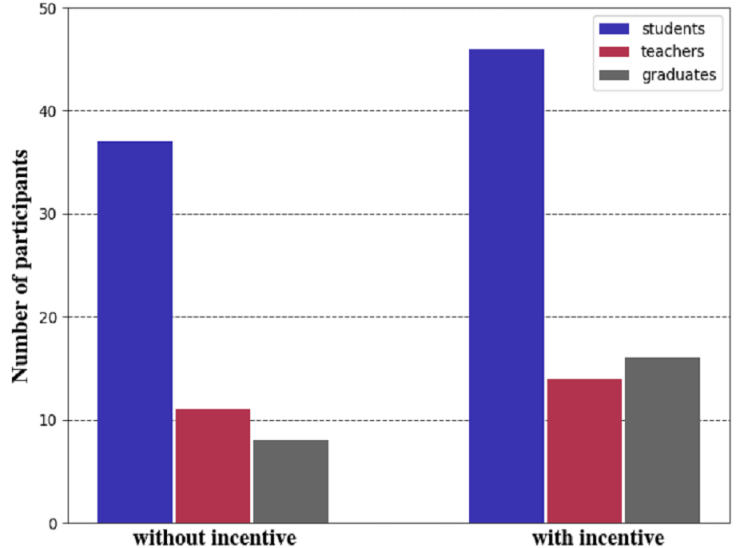

Fig. 6: Comparison of the average number of participatants with and without incentives

into the recommendation result of these malicious participants, while causing the honest participants to be penalized. However, this requires the collusion of more than half of the participants, which is difficult to achieve, and the overall benefit of the collusion attackers is compromised when the additional reward obtained does not exceed the benefit obtained by their honest recommendations.

Collusion attack between miners and participants. Such collusion is difficult to achieve because it requires advance negotiation between the participant and the miner, but in the present mechanism miners are randomly assigned and the participant is not sure whether this recommendation will reach the number of node thresholds and thus complete successfully.

Collusion attack between miners and server. Such collusion may reduce the reward of participants to make the system more profitable, but this type of collusion attack requires the majority of miners in the network to reach a consensus with the server, which is difficult to achieve, and the participants will be demotivated if they receive a lower reward, which defeats the original purpose of the incentive design.

\section{3) System Robustness}

The robustness of the Fabric platform. Fabric is an open source consortium blockchain platform, has a number of advantages over building decentralized applications and running smart contracts. First, it is invariant in nature, so users in non-blockchain systems cannot roll back any information in it. Second, it uses cryptography-based hash technology that prevents attackers from spoofing and ensures the security of the system. In addition, the programs running on the fabric platform will never be down and will always run continuously.

The robustness of blockchain. Blockchain-based incentives have an inherent advantage due to the absence of third parties in the blockchain itself. On the one hand, in a centralized system, an attack by a third party would bring down the whole system, but blockchain has no such risk and can mitigate the security problems caused by the presence of a third party, and thus is sufficiently robust. On the other hand, the nodes in the blockchain are all equal, so when some participating nodes in the system are attacked, the other nodes in the system will not

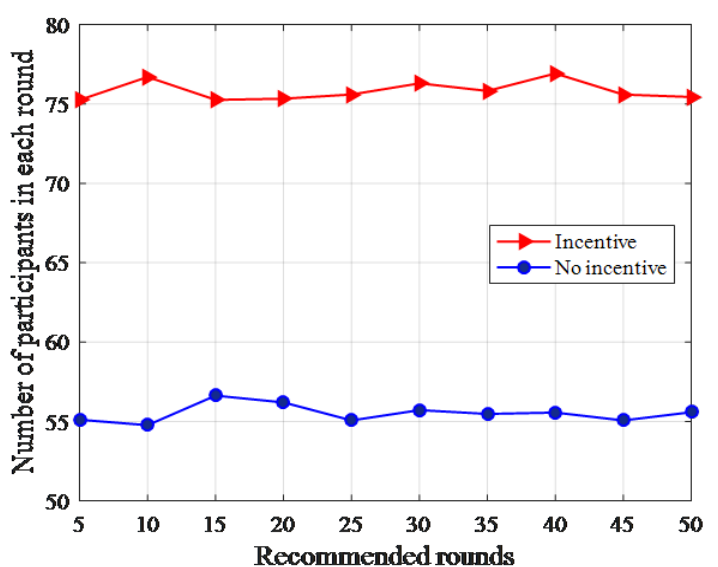

Fig. 7: Comparison of the number of participatants per round with and without incentives

be affected and the system can continue to work. Finally, the structural features of blockchain can also effectively prevent data from being tampered by attackers and can guarantee the correctness and integrity of storaged data.

\section{B. Performance Analysis}

In order to verify the proposed incentive mechanism is superior, we take teacher recommendation as an example for experimental analysis based on the recommendation system in section VI. The simulation analysis is carried out in the following two aspects: firstly, analyze the effectiveness of the incentive mechanism; secondly, compare the overhead of the incentive mechanism with other mechanisms.

In this section, we build a consortium blockchain consisting of two colleges based on the Fabric development environment. In this case, there are 60 participants in each college, including 10 teachers, 30 students, and 20 graduates. They all have access to take part in the teacher recommendation. The system environment configuration is as follows: Windows10 operating system, Intel(R) Core(TM) i5-10210U CPU @ 1.60GHz $2.11 \mathrm{GHz}$ processor, $16 \mathrm{G}$ memory, Matlab R2016a.

\section{1) Analysis of the effectiveness of incentive mechanism}

Firstly, we prove the necessity of introducing the incentive mechanism. For comparison, we simulate the number of participants of the recommendation system with and without an incentive mechanism and conduct 50 rounds of experiments. Fig. 6 shows the change in the average number of 3 types of participants. Before introducing the incentive mechanism, recommendation participants are mainly students. Because in current colleges, recommendation tasks like teacher recommendation are mostly done by students under the supervision of counselors, which lacks the spontaneity of participants. However, after introducing the incentive mechanism, not only students, but some teachers and graduates also actively participated in the recommendation. The number of participatants increased significantly. Actually, the change in the total number of participants per round is reflected in Fig.7.

Next, we prove that our proposed incentive mechanism can improve the accuracy of recommendation results with the 


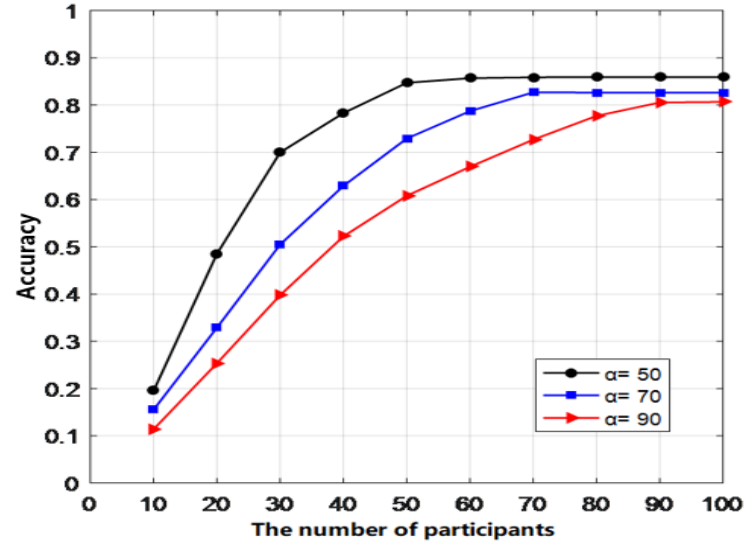

Fig. 8: The effect of the number of participants on the accuracy of recommendation results at different thresholds

increasing number of participatants in the teacher recommendation scenario. On this basis, we also consider the influence of the number of participants threshold on the recommendation results. The threshold $\alpha$ is set to 50,70 , and 90 . Theoretically, when the number of participants reaches the threshold value, more accurate recommendation results will be achieved. The experimental results verify the conclusion. As shown in Fig.8, when the number of participants increases, the accuracy of the recommendation results also increases, and the highest accuracy is achieved when the number of participants is around the threshold. Therefore, the accuracy and credibility of the recommendation results are ensured when the number of participants exceeds the threshold. Our experimental results show that the accuracy of recommendation is close to $90 \%$ when a reasonable number of participants threshold is set in the incentive mechanism.

The experiment result proves that our proposed incentive mechanism is effective and can largely improve the positivity of participatants in the teacher recommendation.

2) Comparative analysis with other incentive mechanisms

After proving the effectiveness of the incentive mechanism, we conduct comparative experiments in the same scenario of teacher recommendation to demonstrate the superiority of our proposed Stackelberg game based incentive mechanism(CRIM). Specifically, we compare the system incentive overheads with the other two incentive mechanisms: the Dynamic price Incentive Mechanism with Virtual Participation credit (RADP-VPC) and the Reputation-based Participatory Incentive Mechanism(RPIM).

The Dynamic price incentive mechanism with virtual participation credit(RADP-VPC): The design goal of this incentive mechanism focuses on retaining participants during recurring reverse auctions while recruiting users who drop out to achieve participant volume maintenance. This mechanism also influences the final reward received by the user by establishing the participation credit variable. Compared to the incentive mechanism designed in this paper, the RADP-VPC mechanism does not consider the factors that influence the value of the user's participation credit comprehensively. However, the mechanism considers a way to minimize the incentive cost

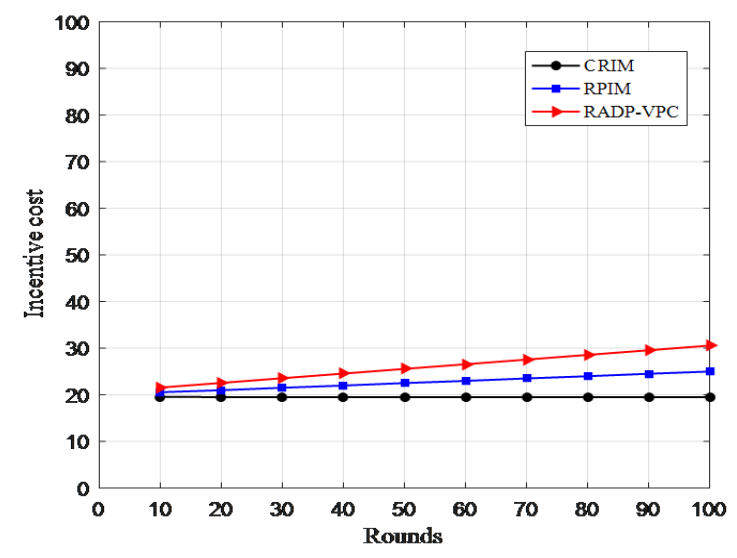

Fig. 9: System incentive overhead without collusion

by preventing it from exploding. Therefore, we compare the incentive mechanism designed in this paper with this incentive mechanism for the incentive cost comparison experiments.

The Reputation-based participatory incentive mechanism(RPIM): This incentive mechanism also adopts game theory for strategic interactions between verifiers in blockchain networks, and rewards users based on their reputation values, which come from the difference between the incentive and the cost of running nodes in the system. However, this incentive mechanism only considers a certain number of rounds of games and lacks consideration of the long-term stability of the system, so we also use it as a comparison experiment for this mechanism.

We set up the comparative experiments under the same teacher recommendation scenario to ensure the fairness and effectiveness. The experiments are conducted in two cases: the case without collusion participation and the case with collusion participation. In the simulation scenario with collusion participation, the number of nodes with normal participation is set to $60 \%$ and the number of collusion participants is set to $40 \%$, and 100 rounds of recommendations are set for each case. Meanwhile, we ensure that there are enough participatants in the recommendation.

The experimental results of the incentive overhead of the system without collusion are shown in Fig.9. As can be seen from the figure, the incentive overhead increases slowly with the increase of the number of recommendation rounds when there is no collusion involved, and the incentive overhead of our proposed mechanism remains basically the same. However, in a comprehensive view, the system incentive overheads of all three mechanisms are relatively small.

The experimental results of the system incentive overhead in the presence of collusion are shown in Fig.10. It can be seen that the system overhead of the CRIM proposed in this paper is relatively smooth, which shows that the incentive mechanism could ensure the credibility of the system and keeps the system incentive overhead low.

Compared with the incentive mechanism proposed in this paper, the system incentive overhead of RADP-VPC increases rapidly, which indicates that this mechanism cannot prevent the occurrence of malicious collusion, and also proves the 


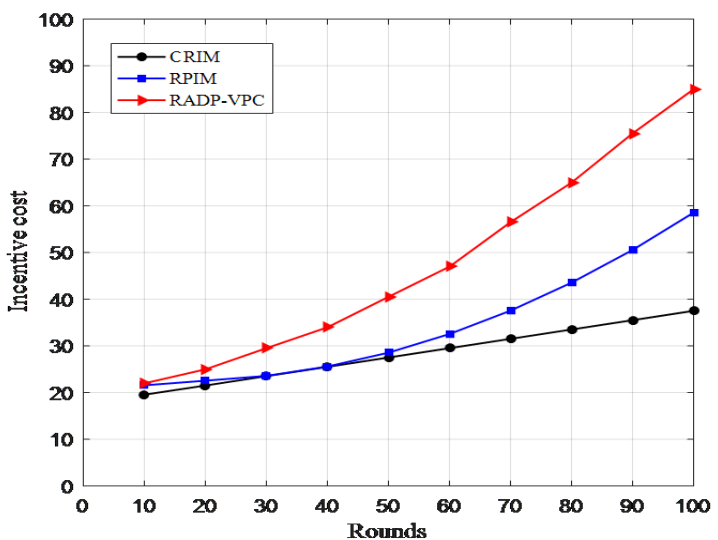

Fig. 10: System incentive overhead with collusion

superiority of introducing Stackelberg game theory in our mechanism, where users and participants not only enhance the credibility of the recommendation results through the strategy selection based on game theory, but also can avoid malicious behaviors in the system to a certain extent.

Meanwhile, the system overhead of RPIM, which is also based on game theory, grows slowly and steadily. Its overhead is between the other two mechanisms, indicating that the incentive mechanism could ensure the credibility of the system to a certain extent. However, since our incentive mechanism considers the long-term participation of users and the longterm stability of the system, the incentive mechanism in this paper has a better performance in the face of malicious collusion.

\section{CONCLUSION}

In this paper, we proposed a reputation incentive mechanism for motivating participatants to take part in recommendation systems and make honest recommendations. First of all, we introduce the system model and the design principles of the incentive mechanism. Next, we adopted the Stackelberg game to design a reputation incentive mechanism, which contains reputation assessment and reward distribution. Moreover, we analyze and prove that the utility between users and recommendation participants can reach a unique Nash equilibrium. Our recommendation system is designed and implemented in the field of education. Finally, through experiments on the scenario of teacher recommendation, we demonstrate that the proposed incentive mechanism is effective and can make recommendation results more accurate. Meanwhile, when compare with the other two incentive mechanisms, our proposed mechanism with Stackelberg game has more superior performance.

\section{ACKNOWLEDGMENT}

The research work was supported by the National Key R\&D Program of China (Grant No. 2020YFB1005500), the Natural Science Foundation of the Jiangsu Higher Education Institutions under (No.20KJB520031), and the Natural Science Foundation of Jiangsu Province under (No.BK20200888).

\section{REFERENCES}

[1] Wang H, Zheng Z, Xie S, et al. Blockchain challenges and opportunities: a survey[J]. International Journal of Web and Grid Services, 2018, 14(4): 352.

[2] Lei A, Cruickshank H, Cao Y, et al. Blockchain-Based Dynamic Key Management for Heterogeneous Intelligent Transportation Systems[J] IEEE Internet of Things Journal, 2017, 4(6): 1832-1843.

[3] Li Y, Hu B. An Iterative Two-Layer Optimization Charging and Discharging Trading Scheme for Electric Vehicle Using Consortium Blockchain[J]. IEEE Transactions on Smart Grid, 2020, 11(3): 2627-2637.

[4] Chen G, Xu B, Lu M, et al. Exploring blockchain technology and its potential applications for education[J]. Smart Learning Environments, 2018, 5(1): 1-10.

[5] Zhao T, Sun G, Feng X, et al. Design of educational resources-oriented fair recommendation system based on consortium blockchain[C]//2020 International Conference on Networking and Network Applications (NaNA). 2020: 448-453.

[6] Yang D, Xue G, Fang X, et al. Crowdsourcing to smartphones: Incentive mechanism design for mobile phone sensing $[\mathrm{C}] / /$ Proceedings of the 18 th annual international conference on Mobile computing and networking. 2012: 173-184.

[7] Kroll J A, Davey I C, Felten E W. The economics of Bitcoin mining, or Bitcoin in the presence of adversaries[C]//Proceedings of WEIS. 2013, 2013: 11

[8] Lee J S, Szymanski B K. Auctions as a dynamic pricing mechanism for e-services[M]//Service Enterprise Integration. Springer, Boston, MA, 2007: 131-156.

[9] Lee J S, Hoh B. Dynamic pricing incentive for participatory sensing[J]. Pervasive and Mobile Computing, 2010, 6(6): 693-708.

[10] Duan L, Kubo T, Sugiyama K, et al. Incentive mechanisms for smartphone collaboration in data acquisition and distributed computing[C]//2012 Proceedings IEEE INFOCOM. IEEE, 2012: 1701-1709.

[11] $\mathrm{Hu} \mathrm{H}, \mathrm{Yu} \mathrm{W}$, Wen G, et al. Reverse group consensus of multi-agent systems in the cooperation-competition network[J]. IEEE Transactions on Circuits and Systems I: Regular Papers, 2016, 63(11): 2036-2047.

[12] Geetha G, Jayakumar C. Implementation of trust and reputation management for free-roaming mobile agent security[J]. IEEE Systems Journal, 2014, 9(2): 556-566.

[13] Zhao Y, Li Y, Mu Q, et al. Secure pub-sub: Blockchain-based fair payment with reputation for reliable cyber physical systems[J]. IEEE Access, 2018, 6: 12295-12303.

[14] Sharples M, Domingue J. The blockchain and kudos: A distributed system for educational record, reputation and reward[C]//European conference on technology enhanced learning. Springer, Cham, 2016: 490-496.

[15] Kantarci B, Glasser P M, Foschini L. Crowdsensing with social networkaided collaborative trust scores[C]//2015 IEEE Global Communications Conference (GLOBECOM). IEEE, 2015: 1-6.

[16] Teutsch J, Reitwießner C. Truebit: a scalable verification solution for blockchains[J]. White Papers, 2018.

[17] He Y, Li H, Cheng X, et al. A blockchain based truthful incentive mechanism for distributed P2P applications[J]. IEEE Access, 2018, 6: 27324-27335.

[18] Ye Q, Zhao T, Sun G, et al. A Recommendation Scheme with Reputation-Based Incentive Mechanism on Consortium Blockchain[C]//2021 International Conference on Networking and Network Applications (NaNA). IEEE, 2021: 313-318.

[19] Nakamoto S. Bitcoin: A peer-to-peer electronic cash system[J]. Decentralized Business Review, 2008: 21260.

[20] Lei A, Cruickshank H, Cao Y, et al. Blockchain-based dynamic key management for heterogeneous intelligent transportation systems [J]. IEEE Internet of Things Journal, 2017, 4(6): 1832-1843.

[21] Li Y, Hu B. An iterative two-layer optimization charging and discharging trading scheme for electric vehicle using consortium blockchain[J]. IEEE Transactions on Smart Grid, 2019, 11(3): 2627-2637.

[22] Zheng Z, Xie S, Dai H N, et al. Blockchain challenges and opportunities: A survey[J]. International Journal of Web and Grid Services, 2018, 14(4): 352-375.

[23] Xue $\mathrm{G}, \mathrm{Xu} \mathrm{J}, \mathrm{Wu} \mathrm{H}$, et al. Incentive mechanism for bitcoin mining pool based on Stackelberg game[C]//International Conference on Science of Cyber Security. Springer, Cham, 2019: 190-198.

[24] $\mathrm{Xu} \mathrm{Z}$, Liu C, Zhang P, et al. URIM: Utility-Oriented RoleCentric Incentive Mechanism Design for Blockchain-Based Crowdsensing[C]//International Conference on Database Systems for Advanced Applications. Springer, Cham, 2021: 358-374. 